\title{
Nanotoxicology: assessment of toxicological properties of nanomaterial
}

\begin{abstract}
Nanotoxicology has been emerging as an important sub discipline of nanotechnology. Nanotoxicology refers to interaction between nanostructure and the biological system with an emphasis on elucidating the relationship between novel physical and chemical properties of nanostructure with induction of toxic responses. Despite the extensive use of nanoparticles there is a very little awareness regarding the toxic effects caused by them. Both in-vitro and in-vivo studies have shown that nanoparticles induce toxicity associated with reactive oxygen species. This mini review gives the briefing of various approaches for assessment of nontoxicity.
\end{abstract}

Keywords: Nanotoxicity, Proteomics, Bioinformatics, Genomics
Volume 6 Issue 2 - 2017

\author{
Sayantan Mukhopadhyay,' Jyoti Nautiyal, ${ }^{2}$ \\ Jyotsana Bhatt, ${ }^{2}$ Sumit Durgapal ${ }^{3}$ \\ 'Department of Pharmaceutics, Shri Guru Ram Rai University, \\ India \\ ${ }^{2}$ Department of Pharmaceutics, Uttarakhand Technical \\ University, India \\ ${ }^{3}$ Department of Pharmaceutical Sciences, Kumaun University, \\ India
}

Correspondence: Sayantan Mukhopdhyay, Department of Pharmaceutics, Division of Pharmaceutical Science, Shri Guru Ram Rai University, India-24800 I, Tel 919760798596; Email sayantan.pharmaceutics@gmail.com

Received: August 28, 2017 | Published:September 06, 2017

\section{Introduction}

With emerging advancement in the Nano based drug delivery system, increasing incidence of Nanotoxicity also observed. Nanomedicines and Nanotoxicity are two sides of the same coin . Nanotoxicology is defined as the study of the interactions of nanostructures with the biological system of human being. Nanotoxicity occurs when biological system induces toxic biological responses due to the interaction with the properties of nanostructures either physical properties, chemical properties or both. Nanostructures include Nanoparticles, Nanotubes, Nano-metals, Nanowire, Nanoballs and Nanocapsules etc.$^{2}$ In simple language, Nanotoxicology is the study of adverse effects which are caused by nanomaterial .

As we know the small size of nanostructures allow them to enter into a particular tissue, cell, organelle and biomolecular structures effectively. The lung is the most important target of nanomaterials but the particle size of the nanostructure is similar to many biological molecules like proteins and antibodies, therefore, it is possible that these nanostructures enter into the vital biological system and cause damage to human health which leads to Nanotoxicity . ${ }^{1}$

For the assessment of nanotoxicity caused due to nanostructures, omics approaches are used. The suffix -ome refers to a totality of some sort, therefore omics refers to a field of study in biological ending in omics such as genomics, proteomics or metabolomics.

\section{Omics approach mainly includes}

a. Bioinformatics: It is generally used to produce predictive and quantitative models of biological pathways.

b. Transcriptomics: To identify changes in gene expression through detection of the number of mRNA copies.

c. Proteomics: It provides information about protein-protein interaction and post translational modification.

d. Metabolomics: It refers to the analysis of the dynamic shift in the production of metabolites of an organism. e. Genomics and epigenomics: Genomics helps to determine the entire DNS sequence of organisms. It generally used in biomedicine to screen for mutation in DNA, in neoplastic disease.

Although exact mechanism underlying the nanotoxicity is not well understood yet. Some mechanisms of toxicological damage have been identified; including reactive oxygen species generation, protein misfolding, membrane perturbation and direct physical damage.$^{5}$ but an important mechanism of nanotoxicity is the generation of reactive oxygen species (ROS). ROS are by products of cellular oxidative metabolism which occurs in mitochondria. ROS include- superoxide anion radicals, hydrogen peroxide, hydroxyl radicals and singlet oxygen (Figure 1).

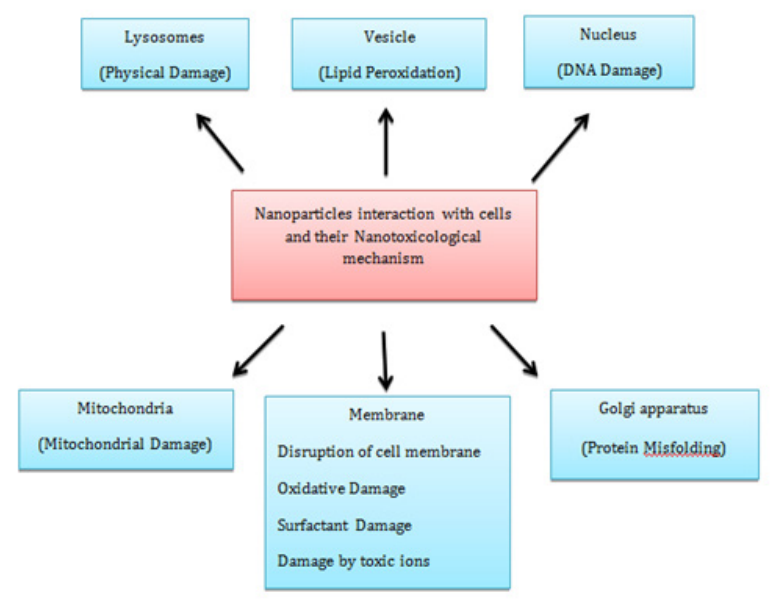

Figure I Nanoparticles interaction with cell \& their Nanotoxical mechanism.

Overproduction of ROS can cause oxidative stress due to which cells failing to maintain normal physiological redox-regulated functions. This failure in maintenance may lead to DNA damage, cytotoxicity, apoptosis, cancer initiation, change in cell motility, unregulated cell signalling. The ROS formation from a particular nanomaterial is dependent on the physical and chemical properties of 
the nonmaterial's such as molecular size, shape, surface area, surface coating, oxidation status, bonded surface species, solubility, and degree of aggregation or agglomeration.$^{6,7}$ Winnik \& Maysinger ${ }^{8}$ observed that quantum dots produced oxidative stress and cause cell damage mediated by ROS.

Akhtar et al. ${ }^{9}$ reported that silica nanoparticle cause cytotoxicity and oxidative stress in a dose dependent manner, due to ROS and lipid peroxidation in the cell membrane. It is reported that Nano-iron oxide, which is super paramagnetic, is cytotoxic and generates ROS and apoptosis.${ }^{10}$ Nanotechnology is a new novel approach for drug delivery which is nowadays very effective but Nanotoxicity is the main concern of nanotechnology. Nanotoxicology as a new discipline should make a remarkable contribution to the development of an effective, sustainable and safe nanotechnology.

\section{Conclusion}

Nanomaterials due to their novel physical and chemical characteristic are widely used in research and medicine. However, these properties may sometimes show calamitous effects in humans. Numerous studies and research have demonstrated that nanoparticles induce reactive oxygen species (ROS) which ultimately prove to be fatal for cells and tissues. Nanotoxicology is thus an assessment of toxicological properties of nanomaterial. Although our knowledge regarding nanotoxicity has increased over last few years still there is a need of evaluating various other aspects for making nanomaterials safe and effective. Hence this subfield of nanotechnology is important for advancement in a wide array of application.

\section{Conflicts of Interest}

The authors declare no conflict of interest

\section{Acknowledgments}

None.

\section{References}

1. Kagan E Valerian, Bayir Hulya, Shvedova A Anna Nanomedicine and Nanotoxicology: Two sides of the same coin Nanomedicine. 2005;1(4):313-316.

2. Fischer HC, Chan WC Nanotoxicity: The growing need for in vivo study. Curr Opin Biotechnol. 2007;18(6):565-571.

3. Arora Sumit, Rajwade M Jyutika, Paknikar M Kishor Nanotoxicology and in vitro studies: The need of the hour. Toxicol App Pharamcol. 2012;258(2):151-165.

4. Costa PM, Fadee B Emerging systems biology approaches in Nanotoxicology: Towards a mechanism-based understanding of nanomaterial hazard and risk. Toxicol Appl Pharamacol. 2015;299:101-111.

5. Elsaesser A, Howard CV Toxicology of Nanoparticles. Adv Drug Deliv Rev. 2012;64(2):129-137.

6. Fu PP, Xia Q, Hwang HM et al. Mechanisms of nanotoxicity: Generation of reactive oxygen species. J Food Drug Anal. 2014;22(1):64-75.

7. Nel A, Xia T, Madler L, Li N Toxic potential of materials at the nanolevel. Science. 2006;311(5761):622-627.

8. Winnik FM, Maysinger D Quantum dot cytotoxicity and ways to reduce it. Acc Chem Res. 2013;46(3):672-680.

9. Akhtar MJ, Ahamed M, Kumar S et al. Nanotoxicity of pure silica mediated through oxidant generation rather than glutathione depletion in human lung epithelial cells. Toxicology. 2010;276(2):95-102.

10. Liu Y, Li X, Bao S et al. Plastic protein microarray to investigate the molecular pathways of magnetic nanoparticle- induced nanotoxicity. Nanotechnology. 2013;24(17):175501. 